\title{
La empatía como herramienta del cuidado enfermero en servicios de oncología pediátrica
}

\section{Empathy as a tool of nursing care in pediatric oncology services}

\author{
María Martos Enriquea, Teresa Galiana Camachoa, M $^{\mathrm{a}}$ Isabel León Latorrea \\ a Facultad Ciencias de la Salud, Universidad de Almería, España
}

\section{Resumen}

Introducción: Los efectos adversos asociados al cáncer generan una serie de cambios biopsicosociales que alteran la vida del niño y su familia. La enfermera dispone de una serie de habilidades, entre las que destaca la empatía, que le permiten garantizar una atención adaptada a las necesidades del niño y su familia. Objetivos: Mostrar los beneficios del uso de la empatía por parte de los enfermeros en el cuidado de los niños oncológicos, valorar la utilidad de la empatía en el trato con las familias e identificar las barreras con las que se encuentran los enfermeros al aplicar la empatía. Metodología: Se realizó una búsqueda bibliográfica en las bases de datos Pubmed, LILACS, ScienceDirect, Scielo y MedlineProquest. Resultados/Discusión: Los estudios revisados mostraron que el uso de la empatía por parte de los enfermeros de oncología pediátrica aportaba notables beneficios en el cuidado del niño y su familia. Se identificaron también una serie de barreras en su aplicación, entre las que destacaban la confusión entre empatizar y simpatizar, la ausencia de habilidades de autorregulación emocional y la falta de experiencia. Conclusión: El uso de la empatía aporta a las enfermeras de oncología pediátrica la capacidad de establecer relaciones basadas en la comprensión y la confianza, permitiendo una atención más humanizada.

Palabras clave: empatía; niño; cáncer; enfermería; enfermería de oncología pediátrica; servicios de oncología pediátrica; cuidados enfermeros.

\begin{abstract}
Introduction: The adverse effects associated with cancer generate a series of biopsychosocial changes which alter the life of the child and his family. The nurse has a series of skills, among which empathy stands out, which allow her to guarantee a care adapted to the needs of the child and his family. Objectives: To show the benefits of the use of empathy by nurses in the care of oncological children, to assess the usefulness of empathy in dealing with families and to identify the barriers that nurses encounter when applying empathy. Methodology: A bibliographic search was conducted in the databases Pubmed, LILACS, ScienceDirect, Scielo and MedlineProquest. Results/Discussion: The studies reviewed showed that the use of empathy by pediatric oncology nurses had significant benefits in the care of the child and family. A number of barriers to its application were also identified, including confusion between empathizing and sympathizing, lack of emotional self-regulatory skills, and lack of experience. Conclusion: The use of empathy gives paediatric oncology nurses the ability to establish relationships based on understanding and trust, allowing for more humanized care.
\end{abstract}

Keywords: empathy; child; cancer; nursing; pediatric oncology nursing; pediatric oncology services; nursing care. 


\section{Introducción}

$\square$ término "cáncer infantil" hace referencia a los distintos tipos de tumores malignos que pueden aparecer en los niños antes de cumplir los 15 años. Entre ellos destacan: la leucemia, los linfomas y los tumores del sistema nervioso central (OMS, 2015). Si bien la tasa de incidencia del cáncer infantil es baja (2-3\% del total de nuevos casos de neoplasias diagnosticadas), durante las últimas décadas ha ido adquiriendo una relevancia creciente, debido a los avances producidos en las técnicas de diagnóstico y tratamiento, así como la disminución de la mortalidad por otras patologías (Amador, Gomes, Coutinho, Costa \& Collet, 2011; Astorino, Kuchinski, Tirapelli, Nakashima \& Batista, 2011; "OMS," 2015). Esto lo ha convertido en la segunda causa de muerte infantil en los países desarrollados, por debajo de los accidentes infantiles (SEHOP, 2012).

La detección precoz y el inicio inmediato del tratamiento, hacen que la probabilidad de recuperación de estos pacientes sea del $80 \%$, más elevada que en el caso de los adultos (SEHO 2012; OMS, 2015). A pesar del buen pronóstico, las repercusiones negativas asociadas al cáncer generan una situación delicada para el niño y sus familias (Astorino et al., 2011; Santos, Silva, Misko, Poles \& Bousso, 2013; Gouveia, Janvier, Dupuis, Duval \& Sultan, 2017).

Los procedimientos empleados para el diagnóstico y el tratamiento del cáncer suelen ser largos, intensos y dolorosos (Olmstead, Scott, Mayan, Koop \& Reid, 2014). El niño no solo debe afrontar las características propias de esta enfermedad y los efectos secundarios de los diferentes tratamientos, sino que además debe hacer frente a la hospitalización, la soledad y la convalecencia (Sousa et al., 2014). Todos estos cambios provocan una compleja multitud de variables sintomáticas, terapéuticas, emocionales y sociales, que impactan casi de forma determinante en su ritmo de vida (Santos et al., 2013). A esto, también se debe añadir que los niños y sus familias se ven obligados a adaptarse rápidamente a nuevas personas, situaciones y ambientes, lo que altera abruptamente su rutina y dinámica familiar (Silva et al., 2018).

La magnitud del impacto que el cáncer genera sobre la vida de los niños y sus familias, hace necesario que deban ser atendidos por un equipo multidisciplinar, formado por profesionales cuyos esfuerzos se centren en proporcionar una atención integral basada en la recuperación biológica, el bienestar y la calidad de vida del niño, y también de sus familias (Sousa et al., 2014).

En este encuadre resulta necesario destacar el papel activo que el profesional de enfermería desempeña en el cuidado del paciente oncológico pediátrico, basado en el uso de habilidades humanísticas, intuitivas y de relación interpersonal (Astorino et al., 2011; Evangelista et al., 2014; Nunes et al., 2018). En el desempeño de su actividad, la enfermera utiliza técnicas que ayudan a reducir la ansiedad, la depresión y el miedo, facilitan los procesos de comunicación y expresión de sentimientos y emociones, y permiten valorar el estado físico, mental y emocional para signos de alarma que amenacen la salud y la calidad de vida del niño y su familia (Santos et al., 2013). Así mismo conocen y aplican herramientas que favorecen el establecimiento de un vínculo de confianza que permita que tanto el propio niño enfermo como la familia sientan que forman parte del proceso, para así generar una atención que va más allá de las técnicas simples (Amador et al., 2011; Nunes et al., 2018; Santos et al., 2013).

Dentro del conjunto de habilidades a disposición de la enfermería, que contribuyen a la prestación de cuidados humanizados y a la mejora de la condición del niño oncológico, cabe señalar el papel de la empatía (Astorino et al., 2011; Santamaría, García, Sánchez Herrera \& Carrillo, 2015; Silva et al., 2018). La empatía constituye la capacidad de ponerse en el lugar del otro, es decir, la capacidad de comprender las experiencias, preocupaciones y perspectivas de otra persona. Por tanto, no es un sentimiento, sino una actitud de la que se derivan diversos sentimientos (Restrepo \& Restrepo, 2017). Requiere captar al otro sin fusionarse con él, conservando un espacio propio de identidad en donde encontrar el equilibrio entre la hiperempatía, que anularía nuestra capacidad de ayudar frente cualquier vicisitud o enfermedad, y la sensibilidad necesaria para entender y ayudar a los pacientes. La empatía no necesariamente va unida a la simpatía, que es un rasgo de carácter y, por tanto, menos educable. La simpatía involucra emocionalmente a los profesionales con los pacientes, pero puede impedir adquirir la distancia necesaria para la toma de decisiones clínicas y, si es excesiva, llevar incluso al agotamiento y al "burn out" (Martínez González \& Galán, 2018.).

Dentro del concepto de empatía, podemos establecer cuatro tipos (Mateu, Campillo, González \& Gómez, 2010):

-Empatía cognitiva: permite entender y reconocer qué está sintiendo la otra persona, siempre desde el intelecto, nunca desde la emoción propia. En este tipo de empatía no existe, por tanto, ningún reflejo afectivo y puede llegar a ser aprendida mediante imitación social. Se trataría del tipo de empatía previa a las demás.

-Empatía participativa: se trata de la forma de 
empatía más empleada y es la que se produce una vez que el sujeto reconoce el estado del otro participante 0 participantes de la situación, sintoniza con él, pero sin emociones. Podría denominarse una falsa empatía o una empatía obligada.

-Empatía afectiva: en este tipo de empatía existe una reacción afectiva mediante la que existe una identificación profunda con los sentimientos de otra persona de manera que pueden sentirse como propios. En ocasiones, si esta empatía emocional es extrema y la identificación con la otra persona es total, puede llegar a dificultar o impedir una correcta actuación profesional.

-Unión empática: en ella, no es la persona la que empatiza con otra, sino que ambas comparten emociones ante una misma experiencia.

En lo referente a la aplicación de la empatía en el ámbito de actuación de los profesionales de enfermería, así como al resto de los profesionales de la salud, surge el concepto de "Empatía Terapéutica" entendido como: "un proceso interactivo destinado a conocer y comprender a otra persona con el fin de facilitar su desarrollo, su crecimiento personal y su capacidad para resolver sus problemas" (Bermejo, 2012). Se concibe, por tanto, la empatía como un aspecto central para el cambio terapéutico, y como un componente fundamental del proceso curativo (Mateu, Campillo, González \& Gómez, 2010; Bermejo 2012).

La empatía bien aplicada a la práctica, constituye una herramienta imprescindible que otorga a los enfermeros la capacidad de reflexionar y entender el impacto que emociones propias de este proceso, tales como el miedo, la inseguridad o el sufrimiento, ejercen sobre los niños y sus familias (Missouridou, 2017; Nunes et al., 2018; Olmstead et al., 2014; Sousa et al., 2014; Taleghani, Ashouri, Memarzadeh \& Saburi, 2018). Esto facilita el establecimiento de relaciones terapéuticas basadas en la comprensión y la comunicación, permitiendo mejorar la asistencia prestada al generar un ambiente de comodidad y satisfacción para el paciente, además de motivar la práctica profesional (Blomberg, Griffiths, Wengström, May \& Bridges, 2016; Nunes et al., 2018; Taleghani et al., 2018).

Todo lo expuesto anteriormente pone de manifiesto la necesidad de que los enfermeros sean conscientes de la importancia del uso de la empatía en el desarrollo de sus funciones en los servicios de oncología pediátrica. Por ello, con esta revisión se pretende dar a conocer los beneficios que el uso de esta habilidad tiene en el cuidado de los pacientes oncológicos pediátricos y en el establecimiento de relaciones de apoyo con las familias.
Además, también se pretende analizar las principales barreras con las que el personal de enfermería se encuentra a la hora de aplicar la empatía en este servicio.

\section{Objetivos}

\section{Objetivo general}

- Analizar la importancia del uso de la empatía por parte de los enfermeros que trabajan en servicios de oncología pediátrica.

\section{Objetivos específicos}

- Mostrar los beneficios del uso de la empatía por parte de los enfermeros en el cuidado de los niños oncológicos. - Valorar la utilidad de la empatía como herramienta de enfermería en el trato con las familias de los niños ingresados en los servicios de oncología pediátrica.

- Identificar las barreras con las que se encuentran los enfermeros al aplicar la empatía en estos servicios.

\section{Metodología}

Para la elaboración de esta revisión, se realizó una búsqueda bibliográfica entre diciembre de 2018 y febrero de 2019 en las bases de datos: LILACS, PubMed, ScienceDirect, Scielo y Medline Proquest, así como la página web de la OMS y de la Sociedad Española de Hematología y Oncología Pediátrica (SEHOP).

La búsqueda en las páginas LILACS y Scielo se realizó utilizando descriptores en ciencias de la salud (DeCS): empatía, enfermería, niño y cáncer. Para PubMed, ScienceDirect y Medline Proquest, se emplearon los términos MeSH (Medical Subject Headings): empathy y nursing. En estos últimos tres recursos, también se emplearon, además los términos en lenguaje no controlado "pediatrics oncology nursing", "pediatric oncology services", "child", "cancer" y "nursing care". El operador booleano utilizado fue AND.

En la tabla 1 se resume la estrategia de búsqueda empleada en las distintas bases de datos consultadas, así como los resultados obtenidos.

Los criterios de inclusión fueron que todos los documentos estuviesen redactados en español, inglés y portugués, que hiciesen referencia a la empatía y que estuviesen centrados en la enfermería pediátrica oncológica. Se excluyeron todos aquellos artículos anteriores a 2009.

La selección de la documentación empleada en la elaboración de este documento comenzó con una revisión inicial de todos los artículos que se ajustaban a los criterios de búsqueda mediante lectura del título. 
Después se realizó una segunda revisión leyendo los resúmenes. Tras esto se examinaron los artículos completos de aquellos que cumplían con los criterios de la búsqueda y que habían sido seleccionados tras la lectura del título y resumen. Se descartaron todos aquellos documentos duplicados cuya metodología no cumplía criterios de calidad. Dichos criterios fueron analizados siguiendo las pautas de valoración critica de artículos establecidas por el "Evidence-Based Medicine Working Group" (McMaster University, Ontario), a las que se accedieron a través del recurso web "Critical Appraisal Skills programme España" (CASPe). La siguiente tabla muestra cómo se realizó el proceso de selección de artículos (tabla 2).

Tabla 1. Estrategia de búsqueda seguida en esta revisión.

\begin{tabular}{|c|c|c|c|c|}
\hline Bases de Datos & Palabras clave & $\begin{array}{l}\text { Operador } \\
\text { booleano }\end{array}$ & $\begin{array}{l}\text { Cadenas de } \\
\text { Búsqueda }\end{array}$ & $\begin{array}{c}\text { Resultados } \\
\text { obtenidos }\end{array}$ \\
\hline LILACS & $\begin{array}{l}\text { Enfermería, niño, } \\
\text { cáncer, empatía }\end{array}$ & AND & $\begin{array}{c}\text { enfermería AND niño } \\
\text { AND cáncer AND } \\
\text { empatía }\end{array}$ & 7 \\
\hline \multirow[t]{2}{*}{ PubMed } & $\begin{array}{l}\text { Nursing, empathy, } \\
\text { child, cancer }\end{array}$ & AND & $\begin{array}{c}\text { «Empathy»[MeSH } \\
\text { Terms] AND child } \\
\text { AND cancer AND } \\
\text { nursing [MeSH } \\
\text { Terms] }\end{array}$ & 26 \\
\hline & $\begin{array}{l}\text { Empathy, pediatric } \\
\text { oncology nursing }\end{array}$ & AND & $\begin{array}{c}\text { «Empathy»[Mesh] } \\
\text { AND pediatric } \\
\text { oncology nursing }\end{array}$ & 21 \\
\hline ScienceDirect & $\begin{array}{c}\text { Empathy, pediatric } \\
\text { oncology services, } \\
\text { nursing care }\end{array}$ & AND & $\begin{array}{c}\text { Empathy AND } \\
\text { pediatric oncology } \\
\text { services and nursing } \\
\text { care }\end{array}$ & 291 \\
\hline Scielo & $\begin{array}{c}\text { Enfermería, cáncer, } \\
\text { niño }\end{array}$ & AND & $\begin{array}{l}\text { Enfermería AND } \\
\text { cáncer AND niño }\end{array}$ & 1 \\
\hline \multirow[t]{2}{*}{ Medline Proquest } & $\begin{array}{l}\text { Nursing, empathy, } \\
\text { child, cancer }\end{array}$ & AND & $\begin{array}{c}\text { Empathy AND child } \\
\text { AND cancer AND } \\
\text { nursing }\end{array}$ & 4 \\
\hline & $\begin{array}{l}\text { Empathy, pediatric } \\
\text { oncology nursing }\end{array}$ & AND & $\begin{array}{c}\text { Empathy AND } \\
\text { pediatric oncology } \\
\text { nursing }\end{array}$ & 49 \\
\hline Total & & & & 399 \\
\hline
\end{tabular}


Tabla 2. Proceso de selección de artículos.

\begin{tabular}{|c|c|c|c|c|}
\hline \multicolumn{5}{|c|}{$1^{\circ}$ paso } \\
\hline LILACS & PubMed & ScienceDirect & Scielo & Medline Proquest \\
7 & 47 & 291 & 1 & 53 \\
\hline \multicolumn{4}{|c|}{ Total: 399} \\
\hline
\end{tabular}

\begin{tabular}{|c|c|c|c|c|}
\hline \multicolumn{5}{|c|}{$2^{\circ}$ paso } \\
\hline LILACS & PubMed & ScienceDirect & Scielo & Medline Proquest \\
4 & 25 & 55 & 1 & 32 \\
\hline \multicolumn{5}{|c|}{ Total:117 } \\
\hline
\end{tabular}

\begin{tabular}{|c|c|c|c|c|}
\hline \multicolumn{5}{|c|}{$3^{\circ}$ paso } \\
Selección de publicaciones tras lectura de texto completo aplicando criterios de inclusión y exclusión \\
\hline LILACS & PubMed & ScienceDirect & Scielo & Medline Proquest \\
3 & 8 & 20 & 1 & 15 \\
\hline \multicolumn{5}{|c|}{ Total: 47} \\
\hline
\end{tabular}

$$
4^{0} \text { paso }
$$

Critica metodológica de los artículos seleccionados

\begin{tabular}{l|l}
36 adecuada calidad metodológica $\Rightarrow 28$ & 8 no cumplen criterios de calidad
\end{tabular} (sin contar duplicados) 


\section{Resultados y discusión}

Con el fin de facilitar su comprensión, los resultados han sido planteados de forma que vayan dando respuesta a los objetivos planteados con anterioridad:

Beneficios observados del uso de la empatía por parte de los enfermeros en el cuidado de los niños oncológicos

Las enfermeras son unos de los miembros del equipo sanitario que más tiempo pasan en compañía de los niños, lo que favorece el desarrollo de relaciones basadas en la comunicación, la afectividad y la empatía (Nunes et al., 2018). El estudio realizado por Missouridou (2017), muestra que el establecimiento de vínculos basados en la empatía posee una gran importancia en estos servicios, al generar un ambiente cómodo y acogedor para el niño, que permite a las enfermeras ganar su confianza y ayuda a mejorar la calidad de los cuidados proporcionados.

Los efectos psicológicos y emocionales adversos asociados al cáncer y la dificultad para hacerles frente, hacen que los profesionales de enfermería se expongan de forma continua a sentimientos de ira, miedo y sufrimiento. Por ello, el uso de una asistencia empática favorece la mejora del estado de ánimo y el bienestar mental y emocional de los niños hospitalizados, buscando progresos satisfactorios en su salud, lo que facilita la recuperación del paciente y minimiza los perjuicios y los traumas de la hospitalización (Astorino et al 2011).

En la relación con los niños hospitalizados con dolor crónico por cáncer, los profesionales de enfermería utilizan la empatía como estrategia de interacción, ya que favorecen la aproximación, la comprensión y la humanización de la atención, evitando la impersonalidad en las relaciones de cuidado (Silva et al., 2018). Olmstead (2014) argumenta que el uso de esta capacidad de empatía, facilita la elaboración de estrategias de juego y entretenimiento que permiten minimizar las experiencias de dolor asociadas al cáncer y a los procedimientos diagnósticos y terapéuticos a los que son sometidos.

Utilidad de la empatía como herramienta de la enfermera de oncología pediátrica en el trato con los familiares de niños hospitalizados

La difícil situación que se produce desde el momento en el que los niños son diagnosticados de cáncer, hace que las familias experimenten una serie de cambios para los que no están preparados (Cerqueira, Pereira, \& do Céu Barbieri Figueiredo, 2016). Incluso si la familia constituye una piedra angular en la atención pediátrica, los padres necesitan un mayor apoyo para poder manejar la situación. En este sentido, el uso de la empatía y el diálogo por parte de los enfermeros, permite la creación de un vínculo de confianza que otorga a las familias la capacidad de expresar sus sentimientos y emociones (Snaman et al., 2016). La investigación llevada a cabo por Gouveia et al., (2017), ponía de manifiesto que uno de los aspectos mejor valorados por las familias de los niños ingresados en estos servicios era el apoyo emocional proporcionado por las enfermeras, gracias a su capacidad de empatizar con ellos.

El uso de la empatía promovido por los profesionales de enfermería, facilita el establecimiento de un proceso de comunicación abierta, que permite a los familiares participar de forma activa en el proceso de atención del niño (Bai, Harper, Penner, Swanson, \& Santacroce, 2017). El diálogo abierto y la empatía da a las familias la posibilidad de realizar preguntas y resolver dudas, lo que facilita el proceso de toma de decisiones (Sandra \& Lina, 2015).

Barreras en el uso de la empatía encontradas por los enfermeros de los servicios de oncología pediátrica

Las enfermeras de oncología pediátrica son particularmente vulnerables a la angustia emocional. Son responsables de la supervisión del cuidado de los niños y mantienen interacciones cercanas con múltiples pacientes y familias a lo largo del tiempo, muchos de las cuales se enfrentan a diagnósticos que limitan su vida (Boyle \& Bush, 2018). La carga emocional generada por estas situaciones crea un fenómeno conocido como "fatiga por compasión" (Hernández, 2017). Perry, Toffner, Merrick \& Dalton (2011) definieron este concepto como un "cansancio debilitante provocado por la exposición al dolor y sufrimiento de los demás", y lo relacionaron con la disminución de la capacidad de empatizar de las enfermeras y un empeoramiento en la calidad de los cuidados que prestaban. Asociada a este concepto, la principal barrera encontrada a la hora de aplicar la empatía en estos servicios, se encuentra en la dificultad que existe para distinguir la empatía de la simpatía. Para mantener una actitud empática, las enfermeras deben tener la habilidad de identificarse con la situación emocional del paciente, sentir como él, pero también debe tener la habilidad de liberarse de esta identificación y reestablecer la distancia emotiva, hecho que no se cumple si simplemente se limitan a simpatizar con el paciente. El equilibrio entre implicación y retirada emotiva es fundamental para no "quemarse" en el desempeño 
de la profesión y para no trabajar desde una excesiva distancia o frialdad con el enfermo (Restrepo, 2017).

El estudio llevado a cabo por da Silva et al (2018) establecía que, como consecuencia del vínculo establecido entre la enfermera y el niño, debido a su larga permanencia en estos servicios y al constante contacto con el equipo de enfermería, estos profesionales sentían la pérdida del paciente como propia, lo que podía llevarlos a crear barreras emocionales que limitaban su aptitud para empatizar. Este hecho es contrastado por Martínez y Galán (2018), quienes concluyeron que involucrarse demasiado o cruzar los límites profesionales, frustra y desvía a la enfermera del cuidado empático del paciente. Cuando la enfermera es particularmente sensible a empatizar con sus pacientes, se encuentra expuesta a compartir sentimientos positivos y también negativos, cuando el sufrimiento es el sentimiento dominante puede caer en lo que se denomina "sufrimiento empático", lo que significa padecer un sufrimiento referido, esto sucede cuando no tiene las habilidades necesarias de autorregulación emocional (Cardona \& García, 2010).

La falta de experiencia y de capacitación formal, constituyen otra barrera en la atención basada en la empatía. Las enfermeras jóvenes que han empezado recientemente su práctica profesional en los servicios de oncología pediátrica, suelen recibir programas de orientación de escasa duración y esa falta de conocimientos puede afectar de manera negativa a la confianza que tienen en sí mismos para empatizar con los pacientes (Taleghani et al., 2018). Además, la poca experiencia que estos profesionales poseen puede llevarles a vivir las relaciones con sus pacientes más como simpatía o amistad, que como empatía. No saber dónde están los límites entre su yo y el del otro, cayendo en una relación de dependencia, de sentirse imprescindible de los pacientes, hasta el punto de no tener vida propia ni permitirse reservarse tiempo para sus propias necesidades tanto físicas, como sociales, emocionales como espirituales (Cibanal et al., 2001).

\section{Conclusiones}

La empatía constituye una herramienta fundamental en el quehacer enfermero de los servicios de oncología pediátrica, pues permite que estos profesionales puedan percibir de forma más clara las nuevas necesidades y requerimientos que tanto los niños como sus familiares, experimentan tras producirse el diagnóstico de cáncer. Que enfermería conozca qué es la empatía y cómo emplearla adecuadamente no solo permitirá el establecimiento de relaciones basadas en la confianza y la comprensión, sino que también les permitirá prestar un cuidado más adecuado al dificultar la aparición de fenómenos tales como la fatiga por compasión, el "burn out" o el distanciamiento emocional que puedan llegar a experimentar.

\section{Referencias Bibliográficas}

Amador, D. D., Gomes, I. P., Coutinho, S. E. D., Costa, T. N. A., \& Collet, N. (2011). Concepção dos enfermeiros acerca da capacitação no cuidado à criança com câncer. Enfermagem, 20(1),94-101. https://doi. org/10.1590/S0104-07072011000100011

Astorino, O., Kuchinski, F. B., Tirapelli, A. B. B., Nakashima, G., \& Batista, N. N. L. de A. L. (2011). Analise histologica do papo de pombo (Columba livia Linaeus, 1758). Journal Health Science Institute, 29(2), 106-109.

Bai, J., Harper, F., Penner, L., Swanson, K., \& Santacroce, S. (2017). Parents' Verbal and Nonverbal Caring Behaviors and Child Distress During Cancer-Related Port Access Procedures: A Time-Window Sequential Analysis. Oncology Nursing Forum, 44(6), 675687. https://doi.org/10.1188/17.ONF.675-687

Bermejo, J.C. (2012). Empatía terapéutica y compasión. Revista Chilena de Endocrinología y Diabetes, 5(2), 93-93.

Blomberg, K., Griffiths, P., Wengström, Y., May, C., \& Bridges, J. (2016). Interventions for compassionate nursing care: A systematic review. International Journal of Nursing Studies, 62, 137-155. https:// doi.org/10.1016/J.IJNURSTU.2016.07.009

Boyle, D. A., \& Bush, N. J. (2018). Reflections on the Emotional Hazards of Pediatric Oncology Nursing: Four Decades of Perspectives and Potential. Journal of Pediatric Nursing, 40, 63-73. https:// doi.org/10.1016/J.PEDN.2018.03.007

Cardona Torres, L. M., \& García Campos, M. de L. (2010). La empatía, un sentimiento necesario en la relación enfermera-paciente. Desarrollo Científico de Enfermería, 18(3), 120-124.

Cerqueira, C., Pereira, F., \& do Céu Barbieri Figueiredo, M. (2016). Patterns of Response in Parents of Children With Cancer: An Integrative Review. Oncology Nursing Forum, 43(2), 43-55. https:// doi.org/10.1188/16.ONF.E43-E55

Cibanal Juan, L., Siles González, J., Arce Sánchez, M. del C., Domínguez Santamaría, J. M., Vizcaya-Moreno, 
M. F., \& Gabaldón Bravo, E. M. (2001). La relación de ayuda es vivificante, no quema. Cultura de Los Cuidados, 5(10), 88-99. https://doi.org/10.14198/ cuid.2001.10.12

Gouveia, L., Janvier, A., Dupuis, F., Duval, M., \& Sultan, S. (2017). Comparing two types of perspective taking as strategies for detecting distress amongst parents of children with cancer: A randomised trial. Plos one, 12(4), 1-15. https://doi.org/10.1371/journal. pone. 0175342

Hernández García, M. del C. (2017). Fatiga por compasión entre profesionales sanitarios de oncología y cuidados paliativos. Psicooncología, 14(1), 53-70. https://doi.org/10.5209/PSIC.55811

Martínez González, C., \& Galán, I. R. (2018). La empatía, elemento clave del currículo oculto. Formación Activa de Pediatría en Atennción Primaria, 11(4),189-190. Retrieved from www.fapap.es

Mateu, C., Campillo, C., González, R.,\& Gómez, O. (2010). La empatía psicoterapéutica y su evaluación: Una revisión. Revista de Psicopatología y Psicología Clínica, 15(1),1-18.

Missouridou, E. (2017). Secondary Posttraumatic Stress and Nurses's Emotional Responses to Patient's Trauma. Journal of Trauma Nursing, 24(2), 110 115. https://doi.org/10.1097/JTN.0000000000000274

Nunes, C. F., Silva, L. F., da Santo, F. H. E., Góes, F. G. B., Moraes, J. R. M. M., (2018). Musical dynamics in the sensitization of nursing students in the face of palliative care in pediatric oncology. Escola Anna Nery, 22(4), 13-18. https://doi.org/10.1590/21779465-ean-2017-0448

Olmstead, D. L., Scott, S. D., Mayan, M., Koop, P. M., \& Reid, K. (2014). Influences shaping nurses' use of distraction for children's procedural pain. Journal for Specialists in Pediatric Nursing, 19(2), 162-171. https://doi.org/10.1111/jspn.12067

Organización Mundial de la Salud. (2015). Preguntas frecuentes sobre el cáncer infantil. World Health Organization.

Perry, B., Toffner, G., Merrick, T., \& Dalton, J. (2011). An exploration of the experience of compassion fatigue in clinical oncology nurses. C Revue Canadienne de Nursing Oncologique, 21(2), 91-105.

Sandra, V.S., \& Lina, Z.S. (2015). Factores relacionados con la depresión en pacientes pediátricos con cáncer y participación de la enfermera en su detección.
Duazary,12(2),164-173. Retrieved from https:// dialnet.unirioja.es/servlet/articulo?codigo $=5156559$

Santamaría, N. P., García, L. E., Sánchez Herrera, B., \& Carrillo, G. M. (2015). Percepción del cuidado de enfermería dado a los pacientes con cáncer hospitalizados. Revista Latinoamericana de Bioética, 16(30),104-128. https://doi.org/10.18359/ rlbi.1443

Santos, M. R. dos, Silva, L., Misko, M. D., Poles, K., \& Bousso, R. S. (2013). Desvelando o cuidado humanizado: percepções de enfermeiros em oncologia pediátrica. Texto \& Contexto Enfermagem, 22(3), 646-653. https://doi. org/10.1590/S0104-07072013000300010

SEHOP. (2019). Sociedad Española de Hematología y Oncología Pediátrica.

Silva, C. M. M., Silva, M. P. C., Ferreira, D. de O., Amaral, J. B. do, Gonçalves, J. R. L., \& Contim, D. (2018). Revista de Enfermagem e Atenção à Saúde (2),83-94.

Silva, T. P. da, Leite, J. L., Stinson, J., Lalloo, C., Silva, Í. R., Jibb, L., \& Jibb, L. (2018). Estratégias de ação e interação para o cuidado à criança hospitalizada com dor oncológica crônica. Enfermagem, 27(4), 20-27. https://doi.org/10.1590/0104-07072018003990017

Snaman, J. M., Torres, C., Duffy, B., Levine, D. R., Gibson, D. V., \& Baker, J. N. (2016). Parental Perspectives of Communication at the End of Life at a Pediatric Oncology Institution. Journal of Palliative Medicine, 19(3), 326-332. https://doi.org/10.1089/ jpm.2015.0253

Sousa, M. L. X. F. de, Reichert, A. P. da S., Sá, L. D. de, Assolini, F. E. P., Collet, N., Sousa, M. L. X. F., \& Collet, N. (2014). Stepping into a new world: the meaning of sicken for the child with cancer. Enfermagem, 23(2), 391-399. https://doi.org/10.1590/010407072014000710013

Taleghani, F., Ashouri, E., Memarzadeh, M., \& Saburi, M. (2018). Barriers to empathy-based care: oncology nurses' perceptions. International Journal of Health Care Quality Assurance, 31(3), 249-259. https://doi.org/10.1108//JHCQA-12-2016-0185

Triana-Restrepo, M. C., \& Triana Restrepo, M. C. (2017). La empatía en la relación enfermera-paciente. Avances en Enfermería, 35(2), 120. https://doi. org/10.15446/av.enferm.v35n2.66941 